\title{
Fractional Parabolic Equations with Generalized Mittag-Leffler Kernels
}

\author{
Abedel-karrem Alomari ${ }^{1}$, Thabet Abdeljawad ${ }^{2}$, D. Baleanu ${ }^{3}$, Khaled Saad ${ }^{4}$, and Qasem \\ Al-Mdallal ${ }^{5}$
}

${ }^{1}$ Yarmouk University

${ }^{2}$ Prince Sultan University

${ }^{3}$ Cankaya University

${ }^{4}$ Najran University

${ }^{5}$ United Arab Emirates University

May 29, 2020

\begin{abstract}
In this paper we apply the fractional integrals with arbitrary order depending on the fractional operators of Riemann type $(\mathrm{ABR})$ and Caputo type (ABC) with kernels of Mittag Lefler in three parameters $\$ \mathrm{E}_{-}\{\backslash \text { alpha, } \backslash \mathrm{mu}\}^{\wedge} \backslash$ gamma $(\backslash \mathrm{lambda}, \mathrm{t}) \$$ for solving the time fractional parabolic nonlinear equation. We utilize these operators with homotopy analysis method (HAM) for constructing the new scheme for generating the successive approximations. This procedure are used successfully on two examples for finding the solutions. The effectiveness and accuracy are verified by clarifying the convergence region in the $\$ \mathrm{~h} \$$-curves as well as by calculating the residual error and the results were accurate. Depending on this results, this treatment can be used to find the approximate solutions to many fractional differential equations.
\end{abstract}

\section{Hosted file}

Paper.pdf available at https://authorea.com/users/327818/articles/455241-fractionalparabolic-equations-with-generalized-mittag-leffler-kernels 
figures/f1a/f1a-eps-converted-to.pdf 
figures/f1b/f1b-eps-converted-to.pdf 
figures/f1c/f1c-eps-converted-to.pdf 
figures/ex1-h/ex1-h-eps-converted-to.pdf 
figures/ex1-r8/ex1-r8-eps-converted-to.pdf 
figures/ex1-r5/ex1-r5-eps-converted-to.pdf 
figures/ex1-r2/ex1-r2-eps-converted-to.pdf 
figures/ex1-8/ex1-8-eps-converted-to.pdf 
figures/ex1-5/ex1-5-eps-converted-to.pdf 
figures/ex1-2/ex1-2-eps-converted-to.pdf 
figures/ex1-r8e/ex1-r8e-eps-converted-to.pdf 
figures/ex1-r5e/ex1-r5e-eps-converted-to.pdf 
figures/ex1-r2e/ex1-r2e-eps-converted-to.pdf 
figures/ex2-h/ex2-h-eps-converted-to.pdf 
figures/ex2-r9/ex2-r9-eps-converted-to.pdf 
figures/ex2-r5/ex2-r5-eps-converted-to.pdf 
figures/ex2-r9e/ex2-r9e-eps-converted-to.pdf 
figures/ex2-r5e/ex2-r5e-eps-converted-to.pdf 
figures/ex2-exact/ex2-exact-eps-converted-to.pdf 
figures/ex2-9/ex2-9-eps-converted-to.pdf 
figures/ex2-5/ex2-5-eps-converted-to.pdf 